\title{
Impact of preoperative depression on 2-year clinical outcomes following adult spinal deformity surgery: the importance of risk stratification based on type of psychological distress
}

\author{
Alexander A. Theologis, MD, ${ }^{1}$ Tamir Ailon, MD, MPH, ${ }^{2}$ Justin K. Scheer, BS, ${ }^{3}$ \\ Justin S. Smith, MD, PhD, ${ }^{2}$ Christopher I. Shaffrey, MD, ${ }^{2}$ Shay Bess, MD, ${ }^{4}$ Munish Gupta, MD, ${ }^{5}$ \\ Eric 0. Klineberg, MD, ${ }^{5}$ Khaled Kebaish, MD, ${ }^{6}$ Frank Schwab, MD, ${ }^{7}$ Virginie Lafage, PhD, \\ Douglas Burton, MD, ${ }^{8}$ Robert Hart, MD, ${ }^{9}$ Christopher P. Ames, MD, ${ }^{10}$ and the \\ International Spine Study Group
}

${ }^{1}$ Departments of Orthopaedic Surgery and ${ }^{10}$ Neurosurgery, University of California, San Francisco, California; ${ }^{2}$ Department of Neurosurgery, University of Virginia Medical Center, Charlottesville, Virginia; ${ }^{3}$ School of Medicine, University of California, San Diego School of Medicine, San Diego, California; ${ }^{2}$ Department of Orthopaedic Surgery, Rocky Mountain Hospital for Children, Denver, Colorado; ${ }^{5}$ Department of Orthopedic Surgery, California, Davis, Sacramento, California; ${ }^{6}$ Department of Orthopedic Surgery, Johns Hopkins University School of Medicine, Baltimore, Maryland; ' Department of Orthopedic Surgery, NYU Hospital for Joint Diseases, New York, New York; ${ }^{8}$ Department of Orthopedic Surgery, University of Kansas Medical Center, Kansas City, Kansas; and ${ }^{9}$ Department of Orthopedic Surgery, Oregon Health Sciences University, Portland, Oregon

OBJECTIVE The objective of this study was to isolate whether the effect of a baseline clinical history of depression on outcome is independent of associated physical disability and to evaluate which mental health screening tool has the most utility in determining 2-year clinical outcomes after adult spinal deformity (ASD) surgery.

METHODS Consecutively enrolled patients with ASD in a prospective, multicenter ASD database who underwent surgical intervention with a minimum 2-year follow-up were retrospectively reviewed. A subset of patients who completed the Distress and Risk Assessment Method (DRAM) was also analyzed. The effects of categorical baseline depression and DRAM classification on the Oswestry Disability Index (ODI), SF-36, and Scoliosis Research Society questionnaire (SRS22r) were assessed using univariate and multivariate linear regression analyses. The probability of achieving $\geq 1$ minimal clinically important difference (MCID) on the ODI based on the DRAM's Modified Somatic Perceptions Questionnaire (MSPQ) score was estimated.

RESULTS Of 267 patients, 66 (24.7\%) had self-reported preoperative depression. Patients with baseline depression had significantly more preoperative back pain, greater BMI and Charlson Comorbidity Indices, higher ODIs, and lower SRS-22r and SF-36 Physical/Mental Component Summary (PCS/MCS) scores compared with those without self-reported baseline depression. They also had more severe regional and global sagittal malalignment. After adjusting for these differences, preoperative depression did not impact 2-year ODI, PCS/MCS, or SRS-22r totals $(p>0.05)$. Compared with those in the "normal" DRAM category, "distressed somatics" $(n=11)$ had higher ODI (+23.5 points), lower PCS $(-10.9)$, SRS-22r activity (-0.9), and SRS-22r total $(-0.8)$ scores $(p \leq 0.01)$, while "distressed depressives" $(n=25)$ had lower PCS (-8.4) and SRS-22r total $(-0.5)$ scores $(p<0.05)$. After adjusting for important covariates, each additional point on the baseline MSPQ was associated with a 0.8 -point increase in 2-year ODI $(p=0.03)$. The probability of improving by at least $1 \mathrm{MCID}$ in 2-year ODI ranged from $77 \%$ to $21 \%$ for MSPQ scores $0-20$, respectively.

CONCLUSIONS A baseline clinical history of depression does not correlate with worse 2-year outcomes after ASD surgery after adjusting for baseline differences in comorbidities, health-related quality of life, and spinal deformity sever-

ABBREVIATIONS ASA = American Society of Anesthesiologists; $\mathrm{ASD}=$ adult spinal deformity; $\mathrm{BMI}=$ body mass index; $\mathrm{CCl}=\mathrm{Charlson}$ Comorbidity Index; $\mathrm{DRAM}=$ Distress and Risk Assessment Method; EBL = estimated blood loss; HRQOL = health-related quality of life; LOS = length of stay; MCID = minimal clinically important difference; MCS = Mental Component Summary of the SF-36; MSPQ = Modified Somatic Perceptions Questionnaire; mZDI = Modified Zung Depression Index; ODI = Oswestry Disability Index; PCS = Physical Component Summary of the SF-36; PI-LL = pelvic incidence-lumbar lordosis; PT = pelvic tilt; SRS-22r = Scoliosis Research Society; SVA = sagittal vertical axis; TK = thoracic kyphosis; ZDS = Zung Self-Rating Depression Scale.

SUBMITTED August 16, 2015. ACCEPTED February 25, 2016.

INCLUDE WHEN CITING Published online May 6, 2016; DOI: 10.3171/2016.2.SPINE15980. 
ity. Conversely, DRAM improved risk stratification of patient subgroups predisposed to achieving suboptimal surgical outcomes. The DRAM's MSPQ was more predictive than MCS and SRS mental domain for 2-year outcomes and may be a valuable tool for surgical screening.

http://thejns.org/doi/abs/10.3171/2016.2.SPINE15980

KEY WORDS adult spinal deformity; depression; health-related quality of life; psychological distress; Distress and Risk Assessment Method; risk stratification

A DULT spinal deformity (ASD) is associated with marked physical and mental impairment. ${ }^{10} \mathrm{Al}-$ though evidence exists indicating that surgical treatment improves the quality of life of patients with ASD that causes pain and disability, ${ }^{18}$ certain concomitant preoperative medical comorbidities and mental health disorders differentially affect the success of surgical intervention. ${ }^{16,22}$ For example, prior work from a large population of patients with ASD demonstrated a significantly higher proportion of patients with a history of baseline depression in the group identified as having the "worst" clinical outcomes at 2 years than in the group who had the "best" 2 -year outcomes. ${ }^{22}$ However, the extent to which depression represents a primary psychiatric condition versus a reactive depression due to pain and disability from spinal deformity is poorly understood. Distinguishing between these 2 phenomena is important for maximizing the accuracy and efficiency of preoperative counseling, providing properly informed consent, and optimizing outcomes postoperatively.

Currently available mental health screening metrics in patients with spinal disorders include, but are not limited to, a baseline clinical history of depression, the Mental Component Summary of the SF-36 (MCS), ${ }^{27}$ the "mental" domain of the Scoliosis Research Society questionnaire (SRS-22r), ${ }^{5}$ and the Distress and Risk Assessment Method (DRAM) questionnaire. The DRAM questionnaire categorizes patients into 4 groups based on the evaluation of depression and somatic awareness using the modified Zung Depression Index (mZDI) and the Modified Somatic Perceptions Questionnaire (MSPQ), respectively. ${ }^{14}$ As such, the DRAM was designed to offer physicians a simple technique to screen for psychological distress and the potential need for further comprehensive mental health evaluation. ${ }^{8}$ In regards to spine surgery, the DRAM was initially used in patients with chronic lowback pain; scores corresponding to greater psychological distress were associated with worsening pain and disability postoperatively. ${ }^{14}$ Significant associations have also been found between psychological distress, as assessed by the DRAM, and patient satisfaction and clinical outcomes after nondeformity spine surgery.,2,6,7,14 Because no previous study has evaluated the DRAM in the setting of ASD, we hypothesized that the DRAM may offer improved risk stratification for patients with ASD undergoing surgical intervention as compared with the aforementioned alternative screening metrics.

The objectives of this study were 2-fold: 1) to determine whether the effect of a baseline clinical history of depression on outcome is independent of associated physical disability, and 2) to evaluate which mental health screening tool has the most utility in determining 2-year clinical outcomes after ASD surgery. Our first hypothesis was that baseline self-reported depression is an independent predictor of worse outcomes 2 years after ASD surgery. Our second hypothesis was that, of the available mental health screening metrics (history of depression, SRS-22r Mental, MCS, and the DRAM), the preoperative DRAM subgrouping is the best predictor of 2-year clinical outcomes for patients with ASD.

\section{Methods}

This study was a retrospective review of a prospective, multicenter database of patients with ASD. Institutional review board approval was obtained at each of the 11 participating centers. The inclusion criteria for the study were age $>18$ years and at least 1 of the following radiographic measures of deformity: scoliosis $\geq 20^{\circ}$, sagittal vertical axis (SVA) $\geq 5 \mathrm{~cm}$, pelvic tilt (PT) $\geq 25^{\circ}$, or thoracic kyphosis $(\mathrm{TK}) \geq 60^{\circ}$. At each center, patients who met the inclusion criteria were approached by their surgeon and/ or clinical research coordinator and asked if he or she was interested in participating in the study. If the patient agreed to participate, he or she was enrolled and baseline patient demographics, radiographic measurements, and health-related quality of life (HRQOL) data (see below) were collected by patient-reported questionnaires and/or interviews with research coordinators and subsequently entered into the central database.

Baseline demographic variables included age, sex, body mass index (BMI), Charlson Comorbidity Index (CCI), American Society of Anesthesiologists (ASA) physical status classification scores, history of prior spine surgery, and a clinical history of depression as self-reported by the patient. The current or past treatment for depression was not assessed. Radiographic measures included baseline and 2-year C7-S1 SVA, TK, PT, pelvic incidence-lumbar lordosis mismatch (PI-LL), and thoracic and lumbar Cobb angles, which were used to classify each deformity using the SRS-Schwab classification. ${ }^{24}$ All radiographic measurements were performed on full-length standing anteroposterior and lateral radiographs using Spineview (ENSAM Laboratory of Biomechanics) at a central location. Preoperative and 2-year HRQOL scores included the Oswestry Disability Index (ODI), ${ }^{9}$ SF-36, ${ }^{27}$ SRS-22r, ${ }^{5}$ and back and leg pain numeric rating scales. The DRAM ${ }^{14}$ questionnaire was also administered to a consecutive subset of patients.

\section{Statistical Analysis}

Frequency distributions and summary statistics were tabulated for all baseline and outcome variables for patients with and without a baseline clinical history of depression and the subset of patients who completed the DRAM. Outcome measures were also analyzed for the 
4 DRAM subgroups based on scores for the mZDI and MSPQ: "normal" (mZDI < 17), "at risk" (mZDI 17-33 and MSPQ < 12), "distressed depressed" (mZDI > 33), "distressed somatic" (mZDI 17-33 and MSPQ > 12). ${ }^{14}$ Categorical variable distributions were compared using the Fisher exact or Pearson chi-square tests, depending on sample and cell sizes. Continuous variables were compared using unpaired t-tests to assess for differences in the distributions of patients classified by categorical variables (baseline depression, DRAM category).

A multivariable linear regression model was created for each continuous outcome (dependent) variable. Baseline demographic, radiographic, and HRQOL variables were included as independent variables, if, on univariable regression, they were significantly associated with the outcome variable of interest at a $\mathrm{p}<0.2$ level. Patient age and sex were included in each model based on an a priori decision. Backward selection was then used to sequentially remove variables that were not significant at a $p<0.05$ level on multivariable regression analysis. Variables were retained in the model, regardless of significance, if they altered the beta coefficient of the main predictor of interest by more than $20 \%$.

A logistic regression model was created to predict the likelihood of achieving $\geq 1$ minimal clinically important difference (MCID) on the ODI $(12.8)^{13}$ versus not. Model building followed the same principles outlined above. From this model, probit regression was used to determine the predicted likelihood of reaching $\geq 1$ MCID on the ODI based on the patients' baseline MSPQ score, adjusted for potential confounders.

\section{Results \\ Demographics}

Of the 1254 consecutive patients with ASD who were offered enrollment at all participating institutions, 608 were enrolled. Of the 608 patients who were enrolled, 365 were eligible for 2-year follow-up. Of those 365 patients, 267 had 2-year follow-up, met inclusion criteria, and were included in this analysis (43 males, 224 females, average age $[ \pm$ SD] $56 \pm 15.2$ years). The 98 patients who were not included were lost to follow-up. Baseline depression as a comorbidity was present in 66 (24.7\%) of the 267 patients. One hundred fourteen patients ( 21 males, 93 females, average age $63.3 \pm 11$ years) completed the DRAM questionnaire and had a minimum of 2-year follow-up. In Tables 1 and 2, preoperative demographics and spinal radiographic measurements for depressed/nondepressed patients and DRAM subgroups are presented, respectively. Compared with patients without depression, patients' with depression had significantly greater preoperative BMI, CCI, and ASA scores, more prior spine operations, and longer operative times (Table 1). Hospital length of stay (LOS) was not significant. Operative estimated blood loss (EBL) was similar between groups (Table 1). Depressed patients also had significantly worse baseline sagittal malalignment, including higher PT, PI-LL, and SVA (Table 1). There were no differences between groups in regards to type of spinal deformity, TK, or thoracic and lumbar Cobb angles (Table 1). Moreover, patients with baseline depression had sig- nificantly greater preoperative back pain and ODI scores, and lower baseline scores on the SF-36 (physical component summary [PCS] and MCS) and 5 SRS-22r domains: activity, pain, appearance, mental, and total (Table 3).

\section{Postsurgery Outcomes}

After surgical intervention, significant improvements occurred in all spinal deformity radiographic parameters and all HRQOL scores for the entire cohort (Tables 1 and 3). Depressed and nondepressed patients had statistically similar absolute improvements in HRQOL scores postoperatively (Table 3). After adjusting for differences in demographics, preoperative radiographic parameters, and baseline HRQOL scores, depressed patients did not differ significantly from nondepressed patients with respect to 2 -year outcome measures. Specifically, their 2-year ODI was 2.81 less $(\mathrm{p}=0.28)$, and their PCS, MCS, and SRS-22r scores were $2.23(\mathrm{p}=0.14), 3.64(\mathrm{p}=0.06)$, and $0.16(\mathrm{p}=$ $0.16)$ lower, respectively.

\section{DRAM}

Subgrouping of the 114 patients who completed the DRAM included "normal" $(\mathrm{n}=22)$, "at risk" $(\mathrm{n}=56)$, "distressed depressive" $(\mathrm{n}=25)$, and "distressed somatic" $(\mathrm{n}=11$; Table 2). The 4 groups were similar in regards to sex; BMI, CCI, and ASA scores; percentage of prior spine surgeries; preoperative SRS-Schwab classification; operative data (i.e., length of operation, EBL); hospital LOS; and all preoperative radiographic spinal deformity parameters (Table 2). HRQOL scores for the DRAM patients are presented in Table 3 . The 4 groups were significantly different in regards to preoperative back pain, ODI, MCS, and 5 SRS-22r domains (activity, pain, appearance, mental, and total). Compared with normal patients, at risk patients and distressed depressives had significantly worse back pain, ODI and MCS scores, and SRS-22r activity, pain, appearance, mental, and total scores. Distressed somatics had significantly worse back pain, MCS scores, and SRS-22r activity, pain, mental, and total scores than normal patients. Compared with at risk patients, distressed depressives had significantly worse ODI and MCS scores, and SRS-22r activity, pain, appearance, mental, and total scores. Distressed somatics had significantly lower MCS scores than at risk patients and significantly worse ODI and MCS scores, and SRS-22r activity, pain, appearance, and total scores than distressed depressives.

After surgery, the at-risk and distressed depressive subgroups experienced significant improvements in all HRQOL outcome measures $(\mathrm{p}<0.05)$. Conversely, the distressed somatic subgroup only had significant improvements in back pain $(\mathrm{p}<0.05)$ and no significant improvements in leg pain or scores for the ODI, MCS/PCS, or SRS domains ( $p>0.05$ ). After surgery, normal patients had significant improvements in all HRQOL domains, except SRS-mental $(\mathrm{p}=0.11)$ and MCS $(\mathrm{p}=0.61)$.

\section{Multivariate Analysis}

Multivariate analysis of the impact of preoperative DRAM category on 2-year HRQOL scores is presented in Table 4. The results demonstrate that, compared with 
TABLE 1. Baseline characteristics, operative data, and spinal radiographic parameters of entire cohort

\begin{tabular}{|c|c|c|c|c|}
\hline Variable & All & Depression & No Depression & p Value \\
\hline No. of patients & 267 & 66 & 201 & NA \\
\hline Mean age \pm SD (yrs) & $56 \pm 15.2$ & $57.9 \pm 10.2$ & $55.4 \pm 16.4$ & 0.83 \\
\hline \multicolumn{5}{|l|}{ Sex } \\
\hline Females & 224 & 56 & 168 & \\
\hline Males & 43 & 10 & 33 & \\
\hline Mean $\mathrm{BMI} \pm \mathrm{SD}$ & $27.2 \pm 6.1$ & $29.5 \pm 6.9$ & $26.5 \pm 5.6$ & $<0.01$ \\
\hline Mean $\mathrm{CCl} \pm \mathrm{SD}$ & $1.5 \pm 1.6$ & $2.1 \pm 1.8$ & $1.3 \pm 1.5$ & $<0.01$ \\
\hline Mean ASA \pm SD & $2.3 \pm 0.7$ & $2.6 \pm 0.5$ & $2.2 \pm 0.7$ & $<0.01$ \\
\hline Prior spine surgery (\%) & $119(44.6)$ & $40(60.6)$ & $79(39.3)$ & $<0.01$ \\
\hline Preop SRS-Schwab coronal curve (\%) & & & & 0.27 \\
\hline Type N & $75(28.5)$ & $19(29.2)$ & $56(28.3)$ & \\
\hline Type T & $15(5.7)$ & $3(4.6)$ & $12(6.1)$ & \\
\hline Type L & $95(36.1)$ & $29(44.6)$ & $66(33.3)$ & \\
\hline Type D & $78(29.7)$ & $14(21.5)$ & $64(32.3)$ & \\
\hline \multicolumn{5}{|l|}{ Mean op data \pm SD } \\
\hline OR time (mins) & $395.8 \pm 132.3$ & $423.7 \pm 110$ & $386.5 \pm 137.9$ & 0.03 \\
\hline $\mathrm{EBL}(\mathrm{ml})$ & $1799.4 \pm 1628.1$ & $1698.8 \pm 1550$ & $1832 \pm 1655.3$ & 0.61 \\
\hline LOS (days) & $8 \pm 4.6$ & $8.9 \pm 6.3$ & $7.7 \pm 3.8$ & 0.07 \\
\hline \multicolumn{5}{|l|}{ Mean radiographic data \pm SD } \\
\hline \multicolumn{5}{|l|}{ SVA (mm) } \\
\hline Preop & $60.8 \pm 76$ & $85.6 \pm 88.2$ & $52.5 \pm 69.9$ & 0.01 \\
\hline 2-yr follow-up & $32.4 \pm 57.3$ & $44.5 \pm 61.6$ & $28.4 \pm 55.5$ & 0.03 \\
\hline \multicolumn{5}{|l|}{$\operatorname{TK}\left({ }^{\circ}\right)$} \\
\hline Preop & $34.3 \pm 18.6$ & $32.1 \pm 19.3$ & $35.1 \pm 18.3$ & 0.16 \\
\hline 2-yr follow-up & $47.5 \pm 17.9$ & $46.5 \pm 19.2$ & $47.9 \pm 17.5$ & 0.67 \\
\hline \multicolumn{5}{|l|}{$\mathrm{PT}\left({ }^{\circ}\right)$} \\
\hline Preop & $23.3 \pm 11.1$ & $26.4 \pm 10.2$ & $22.3 \pm 11.3$ & 0.01 \\
\hline 2-yr follow-up & $21.3 \pm 10.2$ & $23.9 \pm 10.3$ & $20.5 \pm 10$ & 0.01 \\
\hline \multicolumn{5}{|l|}{ PI-LL $\left({ }^{\circ}\right)$} \\
\hline Preop & $14.4 \pm 21.4$ & $21.8 \pm 23$ & $12 \pm 20.3$ & $<0.01$ \\
\hline 2-yr follow-up & $4.1 \pm 15.3$ & $8.8 \pm 17.3$ & $2.5 \pm 14.3$ & $<0.01$ \\
\hline \multicolumn{5}{|l|}{ Thoracic Cobb angle $\left({ }^{\circ}\right)$} \\
\hline Preop & $38.1 \pm 18.5$ & $36.5 \pm 20.3$ & $38.5 \pm 18.1$ & 0.47 \\
\hline 2-yr follow-up & $21.8 \pm 15.8$ & $17.8 \pm 15.7$ & $22.9 \pm 15.8$ & 0.05 \\
\hline \multicolumn{5}{|l|}{ Lumbar Cobb angle $\left({ }^{\circ}\right)$} \\
\hline Preop & $33.7 \pm 17.1$ & $33.7 \pm 16.7$ & $33.8 \pm 17.2$ & 0.95 \\
\hline 2-yr follow-up & $16.4 \pm 12.6$ & $15.2 \pm 11$ & $16.8 \pm 13.1$ & 0.73 \\
\hline
\end{tabular}

OR = operating room; NA = not applicable.

the normal group, the distressed somatic subgroup had significantly higher ODI and lower PCS scores, and SRS$22 \mathrm{r}$ activity and total scores. The distressed depressive subgroup had significantly lower PCS and SRS-22r total scores. The at-risk subgroup did not significantly differ in 2-year HRQOL from the normal group. After adjusting for important covariates, each additional point on the baseline MSPQ was associated with a 0.8 -point increase in 2-year ODI $(\mathrm{p}=0.03$; Table 5$)$. The probability of improving by at least 1 MCID on the 2-year ODI ranged from $77 \%$ to $21 \%$ for MSPQ scores $0-20$, respectively (Table 5 ). Outcomes were not predicted by preoperative radiographic measure- ments and/or other mental health metrics (baseline depression status, SRS-22r Mental domain, or MCS; $p>0.05$ ).

\section{Discussion}

Depression is one of the most common mental health disorders in the US, with an estimated 16 million adults affected in a given year. ${ }^{17}$ Along with other psychiatric disorders, depression significantly influences evaluation, treatment, and clinical outcomes of patients with numerous spinal conditions. ${ }^{1-4,7,15}$ However, the role of mental illness in adults who undergo operations for spinal deformity is not well defined. In this investigation, we evaluated 
TABLE 2. Baseline characteristics, operative data, and spinal radiographic parameters of patients who completed the DRAM questionnaire

\begin{tabular}{|c|c|c|c|c|c|c|}
\hline \multirow[b]{2}{*}{ Variable } & \multirow[b]{2}{*}{ All DRAM } & \multicolumn{4}{|c|}{ DRAM Subgroups } & \multirow[b]{2}{*}{ p Value } \\
\hline & & Normal & At Risk & Distressed Depressive & Distressed Somatic & \\
\hline No. & 114 & 22 & 56 & 25 & 11 & NA \\
\hline Mean age \pm SD (yrs) & $63.3 \pm 11$ & $65.4 \pm 10.4$ & $63.6 \pm 12$ & $62.9 \pm 7.1$ & $58.7 \pm 13.6$ & 0.43 \\
\hline Sex & & & & & & 0.78 \\
\hline Female & 93 & 18 & 44 & 22 & 9 & \\
\hline Male & 21 & 4 & 12 & 3 & 2 & \\
\hline Mean BMI \pm SD & $29.1 \pm 6.6$ & $28 \pm 5.3$ & $29.1 \pm 7.5$ & $28.2 \pm 5$ & $33.5 \pm 6.6$ & 0.16 \\
\hline Mean $\mathrm{CCl} \pm \mathrm{SD}$ & $1.8 \pm 1.7$ & $1.5 \pm 1.2$ & $1.7 \pm 1.7$ & $1.6 \pm 1.6$ & $2.9 \pm 2.1$ & 0.11 \\
\hline Mean ASA scale score \pm SD & $2.5 \pm 0.6$ & $2.2 \pm 0.6$ & $2.5 \pm 0.6$ & $2.5 \pm 0.5$ & $2.5 \pm 0.5$ & 0.22 \\
\hline Prior spine surgery (\%) & $64(56.1)$ & $14(63.6)$ & $26(46.4)$ & $17(68)$ & $7(63.6)$ & 0.99 \\
\hline Preop SRS-Schwab coronal curve (\%) & & & & & & 0.28 \\
\hline Type N & $46(41.4)$ & $6(28.6)$ & $26(47.3)$ & $9(36)$ & $5(50)$ & \\
\hline Type T & $4(3.6)$ & $0(0)$ & $3(5.5)$ & $0(0)$ & $1(10)$ & \\
\hline Type L & $46(41.4)$ & $11(52.4)$ & $19(34.5)$ & $12(48)$ & $4(40)$ & \\
\hline Type D & $15(13.5)$ & $4(19)$ & $7(12.7)$ & $4(16)$ & $0(0)$ & \\
\hline \multicolumn{7}{|l|}{ Mean op data \pm SD } \\
\hline OR time (min) & $398.1 \pm 123$ & $381.1 \pm 129.5$ & $410.3 \pm 126.4$ & $381.7 \pm 120$ & $410.4 \pm 101.8$ & 0.71 \\
\hline $\mathrm{EBL}(\mathrm{ml})$ & $2076.2 \pm 2054.8$ & $1983 \pm 1782.1$ & $1976 \pm 1830$ & $1877.9 \pm 1410.9$ & $3334.4 \pm 4197.6$ & 0.29 \\
\hline LOS (days) & $8.2 \pm 3.9$ & $7.8 \pm 3.7$ & $8.2 \pm 4.4$ & $8.6 \pm 3.7$ & $8.4 \pm 2.3$ & 0.92 \\
\hline \multicolumn{7}{|l|}{ Mean radiographic data \pm SD } \\
\hline \multicolumn{7}{|l|}{ SVA (mm) } \\
\hline Preop & $87.2 \pm 72.9$ & $81.5 \pm 69.7$ & $77.7 \pm 66.7$ & $96.6 \pm 88.3$ & $123.8 \pm 66.1$ & 0.25 \\
\hline 2-yr follow-up & $44.3 \pm 61.4$ & $30.2 \pm 65$ & $48.2 \pm 59.8$ & $45.9 \pm 71$ & $49.7 \pm 38.7$ & 0.47 \\
\hline \multicolumn{7}{|l|}{$\mathrm{TK}\left({ }^{\circ}\right)$} \\
\hline Preop & $34.7 \pm 17.2$ & $-36.2 \pm 16$ & $-34.5 \pm 14.8$ & $-35.7 \pm 21$ & $-30.3 \pm 22.4$ & 0.73 \\
\hline 2-yr follow-up & $51.2 \pm 17.7$ & $-55.1 \pm 15.4$ & $-48.7 \pm 17.1$ & $-53.5 \pm 21.6$ & $-50.6 \pm 16.1$ & 0.34 \\
\hline \multicolumn{7}{|l|}{$\mathrm{PT}\left({ }^{\circ}\right)$} \\
\hline Preop & $26.5 \pm 10.6$ & $27.5 \pm 11.6$ & $25.5 \pm 10.4$ & $26.9 \pm 11.9$ & $28.7 \pm 6.7$ & 0.61 \\
\hline 2-yr follow-up & $22.5 \pm 10.2$ & $25.1 \pm 11.5$ & $21 \pm 10$ & $23.9 \pm 9.9$ & $22.3 \pm 9.4$ & 0.59 \\
\hline \multicolumn{7}{|l|}{$\mathrm{PI}-\mathrm{LL}\left({ }^{\circ}\right)$} \\
\hline Preop & $20.8 \pm 17.6$ & $19.9 \pm 16.3$ & $18.4 \pm 16.2$ & $22.9 \pm 21.1$ & $30.5 \pm 16.2$ & 0.14 \\
\hline 2-yr follow-up & $4.7 \pm 14.5$ & $3.8 \pm 13.8$ & $4.6 \pm 15.7$ & $5.5 \pm 14.3$ & $4.9 \pm 11.6$ & 0.88 \\
\hline \multicolumn{7}{|l|}{ Thoracic Cobb angle $\left({ }^{\circ}\right)$} \\
\hline Preop & $33.9 \pm 17.1$ & $27.4 \pm 11.7$ & $36.8 \pm 20.2$ & $35.8 \pm 12.8$ & $26.4 \pm 8.5$ & 0.54 \\
\hline 2-yr follow-up & $22.7 \pm 19.4$ & $14.1 \pm 12$ & $26.6 \pm 23.8$ & $21.8 \pm 11.5$ & $18.3 \pm 6$ & 0.60 \\
\hline \multicolumn{7}{|l|}{ Lumbar Cobb angle $\left(^{\circ}\right)$} \\
\hline Preop & $29 \pm 12.9$ & $31.4 \pm 16.6$ & $28 \pm 9.7$ & $29.2 \pm 14.9$ & $25.5 \pm 8.3$ & 0.76 \\
\hline 2-yr follow-up & $15.5 \pm 10.5$ & $15.8 \pm 11.3$ & $16.8 \pm 10.9$ & $14 \pm 9.2$ & $11.9 \pm 10.4$ & 0.67 \\
\hline
\end{tabular}

which mental health screening tool has the most utility in determining 2-year clinical outcomes after an ASD operation and sought to determine the extent to which the effect of a baseline clinical history of depression on postoperative outcomes is a consequence of associated physical disability.

We found that patients with ASD and a baseline clinical history of depression had significantly greater pre- and postoperative functional disability and worse pain compared with patients without a baseline clinical history of depression. These findings are consistent with the following sentiment expressed by Vialle et al.: "patients who are depressed and have chronic pain tend to have more intense symptoms, seek more medical care, and have a low satisfaction rate with medical treatment and thus persistently high disability reports." ${ }^{26}$ Consequently, depression was found to be a strong negative predictor of postoperative return-to-work status among a cohort of 2799 patients with workers' compensation who underwent lumbar fusion. ${ }^{4}$ Additionally, Smith et al. found higher rates of preoperative depression in patients who had the "worst" postoperative outcomes (ODI > 30) after ASD surgery compared with those who had the "best" postoperative outcomes (ODI 0-15). ${ }^{22}$ Although patients with a preoperative histo- 
TABLE 3. HRQOL outcome scores

\begin{tabular}{|c|c|c|c|c|c|c|c|c|c|c|}
\hline \multirow[b]{2}{*}{ Variable } & \multirow[b]{2}{*}{$\begin{array}{c}\text { All } \\
(n=267)\end{array}$} & \multirow[b]{2}{*}{$\begin{array}{l}\text { Depression } \\
(n=66)\end{array}$} & \multirow[b]{2}{*}{$\begin{array}{l}\text { No Depression } \\
\quad(n=201)\end{array}$} & \multirow[b]{2}{*}{$\begin{array}{c}p \\
\text { Value }\end{array}$} & \multirow[b]{2}{*}{$\begin{array}{l}\text { DRAM All } \\
(n=114)\end{array}$} & \multicolumn{4}{|c|}{ DRAM Subgroup } & \multirow[b]{2}{*}{$\begin{array}{c}p \\
\text { Value }\end{array}$} \\
\hline & & & & & & Normal & $\begin{array}{l}\text { At } \\
\text { Risk }\end{array}$ & $\begin{array}{l}\text { Distressed } \\
\text { Depressive }\end{array}$ & $\begin{array}{l}\text { Distressed } \\
\text { Somatic }\end{array}$ & \\
\hline \multicolumn{11}{|l|}{ ODI } \\
\hline Preop & $43 \pm 19.5$ & $54.8 \pm 16.9$ & $39.1 \pm 18.7$ & $<0.01$ & $52.1 \pm 13.8$ & $42.7 \pm 12.4$ & $52.3 \pm 12.7$ & $461.3 \pm 12$ & $49.5 \pm 12.6$ & $<0.01$ \\
\hline 2-yr follow-up & $27.4 \pm 20.8$ & $37.4 \pm 20.4$ & $24.1 \pm 20$ & $<0.01$ & $33.2 \pm 21.1$ & $20.5 \pm 15.4$ & $31.4 \pm 18.7$ & $41.8 \pm 23.2$ & $47.6 \pm 22.7$ & $<0.01$ \\
\hline \multicolumn{11}{|l|}{ SF-36 PCS } \\
\hline Preop & $32.5 \pm 10.1$ & $28.2 \pm 7.3$ & $34 \pm 10.5$ & $<0.01$ & $27.8 \pm 7.5$ & $28.1 \pm 9.1$ & $28.1 \pm 9.1$ & $26.6 \pm 5$ & $27.6 \pm 6.5$ & 0.79 \\
\hline 2-yr follow-up & $40.5 \pm 11.6$ & $35.6 \pm 10.7$ & $42.2 \pm 11.4$ & $<0.01$ & $36.7 \pm 10$ & $39.9 \pm 9.1$ & $37.6 \pm 10.7$ & $33.7 \pm 8.3$ & $31.5 \pm 9.2$ & 0.06 \\
\hline \multicolumn{11}{|l|}{ SF-36 MCS } \\
\hline Preop & $44.8 \pm 13.7$ & $35 \pm 12.6$ & $48.1 \pm 12.4$ & $<0.01$ & $42.4 \pm 13.8$ & $56.5 \pm 11$ & $44.4 \pm 9.5$ & $26.8 \pm 8.6$ & $38.5 \pm 11.9$ & $<0.01$ \\
\hline 2-yr follow-up & $49.8 \pm 12.8$ & $42.1 \pm 13.4$ & $52.4 \pm 11.5$ & $<0.01$ & $48.9 \pm 13.2$ & $58.7 \pm 6.8$ & $50.1 \pm 12.6$ & $40.6 \pm 11.4$ & $39.6 \pm 14.6$ & $<0.01$ \\
\hline \multicolumn{11}{|l|}{ SRS activity } \\
\hline Preop & $2.9 \pm 0.9$ & $2.3 \pm 0.7$ & $3.1 \pm 0.9$ & $<0.01$ & $2.5 \pm 0.7$ & $3.1 \pm 0.7$ & $2.6 \pm 0.7$ & $1.9 \pm 0.5$ & $2.5 \pm 0.5$ & $<0.01$ \\
\hline 2-yr follow-up & $3.5 \pm 1$ & $2.9 \pm 1.1$ & $3.7 \pm 1$ & $<0.01$ & $3.2 \pm 1$ & $3.9 \pm 0.8$ & $3.2 \pm 0.9$ & $2.8 \pm 1.1$ & $2.6 \pm 0.9$ & $<0.01$ \\
\hline \multicolumn{11}{|l|}{ SRS pain } \\
\hline Preop & $2.4 \pm 0.9$ & $1.9 \pm 0.6$ & $2.6 \pm 0.8$ & $<0.01$ & $2.1 \pm 0.6$ & $2.6 \pm 0.6$ & $2.1 \pm 0.6$ & $1.7 \pm 0.5$ & $2 \pm 0.5$ & $<0.01$ \\
\hline 2-yr follow-up & $3.4 \pm 1.1$ & $2.9 \pm 1.2$ & $3.6 \pm 1.1$ & $<0.01$ & $3.2 \pm 1.1$ & $3.7 \pm 0.9$ & $3.3 \pm 1.1$ & $2.9 \pm 1.2$ & $2.5 \pm 1.1$ & 0.01 \\
\hline \multicolumn{11}{|l|}{ SRS appearance } \\
\hline Preop & $2.4 \pm 0.8$ & $2.1 \pm 0.8$ & $2.6 \pm 0.7$ & $<0.01$ & $2.3 \pm 0.7$ & $2.5 \pm 0.6$ & $2.3 \pm 0.6$ & $1.7 \pm 0.6$ & $2.4 \pm 0.8$ & $<0.01$ \\
\hline 2-yr follow-up & $3.6 \pm 0.9$ & $3.3 \pm 1$ & $3.8 \pm 0.9$ & $<0.01$ & $3.4 \pm 0.9$ & $4.1 \pm 0.5$ & $3.4 \pm 0.8$ & $3 \pm 1$ & $3 \pm 0.9$ & $<0.01$ \\
\hline \multicolumn{11}{|l|}{ SRS mental } \\
\hline Preop & $3.4 \pm 0.9$ & $2.7 \pm 0.8$ & $3.6 \pm 0.9$ & $<0.01$ & $3.2 \pm 0.9$ & $4.1 \pm 0.7$ & $3.2 \pm 0.7$ & $2.5 \pm 0.7$ & $2.8 \pm 1$ & $<0.01$ \\
\hline 2-yr follow-up & $3.8 \pm 0.9$ & $3.3 \pm 0.9$ & $4 \pm 0.8$ & $<0.01$ & $3.8 \pm 0.9$ & $4.4 \pm 0.6$ & $3.8 \pm 0.8$ & $3.4 \pm 1$ & $3.4 \pm 0.9$ & $<0.01$ \\
\hline \multicolumn{11}{|l|}{ SRS satisfaction } \\
\hline Preop & $2.8 \pm 1.1$ & $2.6 \pm 1.1$ & $2.8 \pm 1$ & 0.06 & $2.9 \pm 1.1$ & $3 \pm 1.3$ & $2.9 \pm 1.1$ & $2.4 \pm 0.8$ & $3.1 \pm 1.4$ & 0.13 \\
\hline 2-yr follow-up & $4.2 \pm 1$ & $4 \pm 1$ & $4.3 \pm 0.9$ & 0.02 & $4.2 \pm 0.9$ & $4.7 \pm 0.6$ & $4.2 \pm 0.9$ & $3.8 \pm 1.1$ & $3.9 \pm 1.2$ & $<0.01$ \\
\hline \multicolumn{11}{|l|}{ SRS total } \\
\hline Preop & $2.8 \pm 0.7$ & $2.3 \pm 0.6$ & $3 \pm 0.6$ & $<0.01$ & $2.6 \pm 0.6$ & $3.1 \pm 0.4$ & $2.6 \pm 0.5$ & $2 \pm 0.4$ & $2.5 \pm 0.5$ & $<0.01$ \\
\hline 2-yr follow-up & $3.7 \pm 0.8$ & $3.2 \pm 0.9$ & $3.8 \pm 0.8$ & $<0.01$ & $3.5 \pm 0.8$ & $4.1 \pm 0.5$ & $3.5 \pm 0.8$ & $3.1 \pm 0.9$ & $3 \pm 0.8$ & $<0.01$ \\
\hline \multicolumn{11}{|l|}{ Back Pain } \\
\hline Preop & $7.2 \pm 2.3$ & $8.1 \pm 1.7$ & $7 \pm 2.4$ & $<0.01$ & $7.8 \pm 2$ & $6.6 \pm 2.4$ & $7.9 \pm 1.7$ & $8 \pm 2$ & $8.7 \pm 1.7$ & 0.02 \\
\hline 2-yr follow-up & $3.6 \pm 3.2$ & $4.5 \pm 3.2$ & $3.4 \pm 3.1$ & $<0.01$ & $4.1 \pm 3.2$ & $3 \pm 2.5$ & $3.9 \pm 3.2$ & $5 \pm 3.4$ & $5.3 \pm 3.7$ & 0.14 \\
\hline \multicolumn{11}{|l|}{ Leg pain } \\
\hline Preop & $4.3 \pm 3.4$ & $5 \pm 3.1$ & $4.1 \pm 3.4$ & 0.07 & $5.5 \pm 3.1$ & $6 \pm 2.8$ & $5 \pm 3.2$ & $5.7 \pm 2.8$ & $6.6 \pm 3.3$ & 0.33 \\
\hline 2-yr follow-up & $2.6 \pm 3$ & $2.9 \pm 3$ & $2.5 \pm 3$ & 0.49 & $3.2 \pm 3$ & $2.1 \pm 2.8$ & $3.2 \pm 2.8$ & $3.5 \pm 3$ & $4.5 \pm 3.4$ & 0.20 \\
\hline \multicolumn{11}{|l|}{ DRAM } \\
\hline \multicolumn{11}{|l|}{$M S P Q$} \\
\hline Preop & NA & NA & NA & NA & $7 \pm 5.1$ & $3.4 \pm 2.8$ & $4.8 \pm 2.9$ & $11.6 \pm 5$ & $14.3 \pm 2$ & $<0.01$ \\
\hline 2-yr follow-up & & & & & $7 \pm 5.5$ & $4 \pm 3.1$ & $5.8 \pm 5.2$ & $10.7 \pm 5.2$ & $9.8 \pm 6.6$ & $<0.01$ \\
\hline \multicolumn{11}{|l|}{ mZDI } \\
\hline Preop & NA & NA & NA & NA & $25.5 \pm 9.9$ & $12.7 \pm 3.3$ & $24.3 \pm 4.4$ & $40.1 \pm 5.4$ & $25 \pm 5$ & $<0.01$ \\
\hline 2-yr follow-up & & & & & $19.9 \pm 11$ & $11 \pm 7.1$ & $19.8 \pm 11$ & $28.4 \pm 9$ & $17.9 \pm 9$ & $<0.01$ \\
\hline
\end{tabular}

ry of depression in our study had significantly worse 2-year HRQOL scores than nondepressed patients after surgery, our observed absolute improvements in HRQOL between depressed and nondepressed groups were similar, which is in contrast to previous studies of nondeformity spinal disorders. ${ }^{2,3,15}$ For example, in a retrospective review of 919 patients who underwent elective lumbar decompression and fusion, Miller et al. found that increased preoperative pain and depression were associated with significantly reduced improvement in postoperative EuroQol-5D scores. ${ }^{15}$ Our conclusion likely differs from those for these previous investigations because our study evaluated patients with ASD, which is a unique pathology with a very different surgical invasiveness than other nondeformity spinal disorders and their respective treatments that have been the focus of previous investigations..$^{2,3,15}$ Furthermore, 2 of our 
TABLE 4. Impact of baseline DRAM category on functional outcome after ASD surgery*

\begin{tabular}{|c|c|c|c|c|c|c|c|c|}
\hline \multirow{2}{*}{$\begin{array}{l}\text { DRAM } \\
\text { Category }\end{array}$} & \multicolumn{2}{|c|}{ ODI } & \multicolumn{2}{|c|}{ PCS } & \multicolumn{2}{|c|}{ SRS-22r Activity } & \multicolumn{2}{|c|}{ SRS-22r Total } \\
\hline & Crude & Adjusted $†$ & Crude & Adjustedf & Crude & Adjusted & Crude & Adjusted§ \\
\hline At risk & $10.9 \rrbracket$ & 4.5 & -2.3 & -1.8 & $-0.6 \pi$ & -0.3 & $-0.6 \pi$ & -0.4 \\
\hline $\begin{array}{l}\text { Distressed } \\
\text { depressive }\end{array}$ & $21.4^{* *}$ & 5.8 & -6.29 & $-8.4 \pi$ & $-1.1^{* *}$ & -0.5 & $-1.0^{* *}$ & $-0.5 \rrbracket$ \\
\hline $\begin{array}{r}\text { Distressed } \\
\text { somatic }\end{array}$ & $27.2^{* *}$ & $23.5 \pi$ & $-8.4 \Uparrow$ & $-10.9 \rrbracket$ & $-1.2^{* *}$ & $-0.9 \rrbracket$ & $-1.1^{* *}$ & $-0.8 \rrbracket$ \\
\hline $\begin{array}{l}\text { * Estimates repr } \\
\text { estimates accour } \\
\dagger \text { ODI also adjus } \\
\ddagger \text { SF-36 PCS als } \\
\S \text { SRS-22r ques } \\
\text { ๆ Significant at } \\
\text { ** Significant at }\end{array}$ & $\begin{array}{l}\text { ent expec } \\
\text { or baselir } \\
d \text { for base } \\
\text { adjusted } \\
\text { nnaire als } \\
0.05 \text {. } \\
0.001 .\end{array}$ & $\begin{array}{l}\text { ncrease or de } \\
\mathrm{DI} \text {, age at the } \\
\text { back pain, le } \\
\text { aseline leg pe } \\
\text { justed for his }\end{array}$ & $\begin{array}{l}\text { o in outc } \\
\text { of surger } \\
\text {, and hist } \\
\mathrm{Ml} \text {, and S } \\
\text { f prior spi }\end{array}$ & $\begin{array}{l}\text { score for eac } \\
\text { x, and additio } \\
\text { f prior spine } \\
\text { nental subsco } \\
\text { urgery. }\end{array}$ & $\begin{array}{l}\text { AM cate } \\
\text { riables }\end{array}$ & $\begin{array}{l}\text { lative to } \\
\text { elow. }\end{array}$ & & up). Adjustec \\
\hline
\end{tabular}

HRQOL outcome measures (SF-36 and SRS-22r) are different from previous studies' outcome measures (EuorQol5D and SF-12), which makes direct comparisons between the studies difficult. Nevertheless, there is a low chance that our null hypothesis (i.e., depression has no impact on 2-year HRQOL scores) would be rejected, as our study's statistical power is $95 \%$, based on a sample size of 267 and a 5-point difference in ODI, which is a more modest effect size compared with the 15-point difference in ODI we noted with our univariate analysis.

A clinical history of depression reflects a mix of severities, treatments, and treatment responses, and likely misses many patients who may have some degree of unrecognized depression. Therefore, when depression is used as a binary classification scheme, important nuances and the severity of a patient's psychological profile may be overlooked. In this study, we found that after adjusting for preoperative differences in demographics, radiographic parameters, and HRQOL scores between depressed and nondepressed patients, preoperative depression did not independently impact 2-year ODI, PCS, MCS, or SRS-22r total scores, suggesting that depression status in this cohort was closely linked to functional disability and pain. In the setting of chronic disease, it is important to distinguish depression that arises secondary to the disease itself from that manifesting as a primary psychiatric condition. This may be best achieved with screening tools that evaluate the severity of depression and concomitant psychiatric illness. To this effect, Alvin et al. ${ }^{3}$ demonstrated that patients with a greater degree of preoperative depression, as assessed by the Patient Health Questionnaire-9, ${ }^{23}$ have lower postoperative quality of life scores compared with those with less severe depression after posterior cervical fusions. ${ }^{3}$ Similarly, Adogwa et al. also found that a higher preoperative Zung Self-Rating Depression Scale (ZDS) score was an independent predictor of lower functional improvement following revision lumbar surgery in elderly patients with symptomatic pseudarthrosis, adjacent-segment disease, or recurrent same-level stenosis. ${ }^{2}$

Although the ZDS is a reliable and valid method to assess depressive symptoms, its utility is limited in patients with pain. ${ }^{25}$ In contrast to the ZDS, the mZDI (one component of the DRAM questionnaire) is more highly associ- ated with pain-related disability than personality traits or hypochondriacal fears..$^{25}$ Because the second component of the DRAM, the MSPQ, assesses heightened somatic awareness ("somatic anxiety") among patients with chronic pain ${ }^{25}$ combined scores from the mZDI and MSPQ have been found to classify risk of poor outcome with considerable accuracy. ${ }^{25}$ In a prospective study of 102 patients who underwent elective lumbar spine surgery, failure to return to work and/or failure to report improvement in pain and functional abilities were predicted by preoperative somatic anxiety and depression, as assessed by the DRAM..$^{25}$ In a separate prospective analysis of patients who underwent anterior cervical decompression and fusion, Peolsson et al. found that a "normal" rating on the DRAM was a significant factor in predicting improvement in postoperative arm pain, neck pain, NDI scores, and general health. ${ }^{19}$ Additionally, Pollock et al. noted that patients classified preoperatively as distressed somatic were the only DRAM subgroup not to have significant improvements in ODI and pain scores after posterior lumbar interbody fusion. ${ }^{20} \mathrm{We}$ also found that the distressed somatic subgroup was the only group not to have significant improvements in ODI, SRS, or SF-36 scores. At-risk and distressed depressive subgroups had significant improvements in back and leg pain as well as all HRQOL scores, although these were attenuated in the distressed depressives relative to the nor-

TABLE 5. Probit model results of impact of MSPQ score on probability of achieving at least $1 \mathrm{MCID}$ improvement on the ODI*

\begin{tabular}{cc}
\hline MSPQ Score & Probability of at Least 1 MCID Improvement in ODI (\%) \\
\hline 0 & 77.3 \\
\hline 3 & 69.8 \\
\hline 6 & 61.3 \\
\hline 9 & 52.3 \\
\hline 12 & 43.1 \\
\hline 15 & 34.3 \\
\hline 20 & 26.3 \\
\hline
\end{tabular}

* Adjusted for baseline SF-36 MCS and PCS scores, back pain, leg pain, BMI, lumbopelvic mismatch (PI-LL), and C7-sacrum SVA. 
mal group. The differences between our DRAM results and results of patients with a clinical history of depression are that the clinical diagnosis group has likely been treated for depression and some of the DRAM patients may have an unrecognized diagnosis without treatment. While we purport that the DRAM may be capturing untreated or poorly treated depression, our data cannot confirm this conjecture, because it does not provide insight into our patients' current or past treatments for depression.

An additional finding of our study was that preoperative DRAM category was the only mental health metric that reliably predicted 2-year HRQOL scores. Comparison of the individual DRAM subgroups demonstrated that the distressed depressive subgroup had lower PCS and SRS22r total scores than normal patients, and the distressed somatic subgroup had significantly higher ODI and lower PCS, SRS activity, and SRS-22r total scores than normal patients had. After adjusting for important covariates, each additional point on the baseline MSPQ was associated with a 0.8 -point increase in 2-year ODI. And the probability of improving by at least 1 MCID in 2-year ODI ranged from $77 \%$ to $21 \%$ for MSPQ scores $0-20$, respectively. This emphasizes the idea that patients with greater somatic anxiety are a particularly vulnerable group that may warrant formal psychological assessment and/or intervention before undergoing an operation for ASD.

Our study should be interpreted within the context of its design and associated limitations. Although we evaluated a relatively large number of patients with ASD, the patients were gathered from 11 heterogeneous institutions, which varied in their enrollment rates $(0.8 \%-18.4 \%)$. As such, there are surgeon-, patient-, and institution-specific factors that likely vary between sites (i.e., surgical techniques, postoperative protocols, cultural factors, etc.), for which we were not able to control and that may introduce bias into our results. Furthermore, sample sizes within each DRAM subgroup were relatively small. However, the large effect size comparing DRAM groups to normal (especially distressed somatic) is fairly compelling. While the DRAM questionnaire ideally would have been available for analysis for all 267 patients in this study, the use of the questionnaire was stopped during the enrollment process at each site. Therefore, our subset of patients who completed the DRAM represents a consecutive group of patients who appear to be a representative sample of the total cohort and not one of the larger groups that selectively chose to complete the DRAM, which decreases bias related to this subgroups' results. Additionally, this investigation only studied patients who underwent operative intervention. The effect of preoperative DRAM category on outcomes after conservative management in adults with spinal deformity is also important to understand, as previous studies have demonstrated that psychological disorders differentially affect treatment outcomes for chronic low-back pain. ${ }^{7,11}$ In a systematic review, Daubs et al. concluded that patients with a personality disorder, more severe depression, and more neuroticism appear to respond more favorably to conservative management, while fusion appears to be better suited for patients without these characteristics. ${ }^{7}$ Our study neither assessed the status of current or past treatments for those who self-reported a clinical history of depression or those who completed the DRAM questionnaire, nor evaluated mental health-specific preoperative interventions, i.e., cognitive behavioral therapy and/or pharmacological therapies and their effects on postoperative outcomes, which has been studied previously in adults with spinal disorders. ${ }^{12,21}$ Despite these limitations, we believe our findings are unique contributions to the growing literature on determinants of outcome after ASD surgery, represent preliminary steps in defining the role for evaluation of mental health in patients with ASD, and highlight the importance of revisiting the DRAM questionnaire for surgical risk stratification for ASD. Ideally, our results should be confirmed in future prospective investigations with larger cohorts and the use of similar clinical outcome tools.

\section{Conclusions}

Categorical baseline depression was strongly associated with worse baseline radiographic deformity and HRQOL measures. However, after adjusting for these factors, preoperative depression did not affect 2-year outcomes after ASD surgery. Conversely, the DRAM improved risk stratification of patient subgroups predisposed to achieving suboptimal surgical outcomes. In particular, the distressed somatic group appears to be uniquely prone to experiencing inferior results. The MSPQ score provides a guideline for estimating the likelihood of improving by at least 1 MCID on the ODI. This information may aid in point of care decision-making and in counseling patients regarding expected outcomes after ASD surgery.

\section{References}

1. Abtahi AM, Brodke DS, Lawrence BD, Zhang C, Spiker WR: Association between patient-reported measures of psychological distress and patient satisfaction scores after spine surgery. J Bone Joint Surg Am 97:824-828, 2015

2. Adogwa O, Parker SL, Shau DN, Mendenhall SK, Aaronson OS, Cheng JS, et al: Preoperative Zung Depression Scale predicts outcome after revision lumbar surgery for adjacent segment disease, recurrent stenosis, and pseudarthrosis. Spine J 12:179-185, 2012

3. Alvin MD, Miller JA, Sundar S, Lockwood M, Lubelski D, Nowacki AS, et al: The impact of preoperative depression on quality of life outcomes after posterior cervical fusion. Spine J 15:79-85, 2015

4. Anderson JT, Haas AR, Percy R, Woods ST, Ahn UM, Ahn NU: Clinical depression is a strong predictor of poor lumbar fusion outcomes among workers' compensation subjects. Spine (Phila Pa 1976) 40:748-756, 2015

5. Asher MA, Lai SM, Glattes RC, Burton DC, Alanay A, Bago J: Refinement of the SRS-22 Health-Related Quality of Life questionnaire function domain. Spine (Phila Pa 1976) 31:593-597, 2006

6. Daubs MD, Hung M, Adams JR, Patel AA, Lawrence BD, Neese AM, et al: Clinical predictors of psychological distress in patients presenting for evaluation of a spinal disorder. Spine J 14:1978-1983, 2014

7. Daubs MD, Norvell DC, McGuire R, Molinari R, Hermsmeyer JT, Fourney DR, et al: Fusion versus nonoperative care for chronic low back pain: do psychological factors affect outcomes? Spine (Phila Pa 1976) 36 (21 Suppl):S96-S109, 2011

8. Daubs MD, Patel AA, Willick SE, Kendall RW, Hansen P, Petron DJ, et al: Clinical impression versus standardized questionnaire: the spinal surgeon's ability to assess psychological distress. J Bone Joint Surg Am 92:2878-2883, 2010 
9. Fairbank JC, Pynsent PB: The Oswestry Disability Index. Spine (Phila Pa 1976) 25:2940-2952, 2000

10. Glassman SD, Bridwell K, Dimar JR, Horton W, Berven S, Schwab F: The impact of positive sagittal balance in adult spinal deformity. Spine (Phila Pa 1976) 30:2024-2029, 2005

11. Hägg O, Fritzell P, Ekselius L, Nordwall A: Predictors of outcome in fusion surgery for chronic low back pain. A report from the Swedish Lumbar Spine Study. Eur Spine J 12:22-33, 2003

12. Hyer L, Scott C, Mullen CM, McKenzie LC, Robinson JS: Randomized double-blind placebo trial of duloxetine in perioperative spine patients. J Opioid Manag 11:147-155, 2015

13. Liu S, Schwab F, Smith JS, Klineberg E, Ames CP, Mundis $\mathrm{G}$, et al: Likelihood of reaching minimal clinically important difference in adult spinal deformity: a comparison of operative and nonoperative treatment. Ochsner J 14:67-77, 2014

14. Main CJ, Wood PL, Hollis S, Spanswick CC, Waddell G: The Distress and Risk Assessment Method. A simple patient classification to identify distress and evaluate the risk of poor outcome. Spine (Phila Pa 1976) 17:42-52, 1992

15. Miller JA, Derakhshan A, Lubelski D, Alvin MD, McGirt MJ, Benzel EC, et al: The impact of preoperative depression on quality of life outcomes after lumbar surgery. Spine J 15:58-64, 2015

16. Mok JM, Cloyd JM, Bradford DS, Hu SS, Deviren V, Smith JA, et al: Reoperation after primary fusion for adult spinal deformity: rate, reason, and timing. Spine (Phila Pa 1976) 34:832-839, 2009

17. National Institutes of Health: Major Depression Among Adults. (http://www.nimh.nih.gov/health/statistics/prevalence/major-depression-among-adults.shtml) [Accessed March 18, 2016]

18. Paulus MC, Kalantar SB, Radcliff K: Cost and value of spinal deformity surgery. Spine (Phila Pa 1976) 39:388-393, 2014

19. Peolsson A, Vavruch L, Öberg B: Predictive factors for arm pain, neck pain, neck specific disability and health after anterior cervical decompression and fusion. Acta Neurochir (Wien) 148:167-173, 2006

20. Pollock R, Lakkol S, Budithi C, Bhatia C, Krishna M: Effect of psychological status on outcome of posterior lumbar interbody fusion surgery. Asian Spine J 6:178-182, 2012

21. Rolving N, Nielsen CV, Christensen FB, Holm R, Bünger CE, Oestergaard LG: Does a preoperative cognitive-behavioral intervention affect disability, pain behavior, pain, and return to work the first year after lumbar spinal fusion surgery? Spine (Phila Pa 1976) 40:593-600, 2015

22. Smith JS, Shaffrey CI, Glassman SD, Carreon LY, Schwab FJ, Lafage V, et al: Clinical and radiographic parameters that distinguish between the best and worst outcomes of scoliosis surgery for adults. Eur Spine J 22:402-410, 2013

23. Spitzer RL, Kroenke K, Williams JB: Validation and utility of a self-report version of PRIME-MD: the PHQ primary care study. Primary Care Evaluation of Mental Disorders. Patient Health Questionnaire. JAMA 282:1737-1744, 1999

24. Terran J, Schwab F, Shaffrey CI, Smith JS, Devos P, Ames CP, et al: The SRS-Schwab adult spinal deformity classification: assessment and clinical correlations based on a prospective operative and nonoperative cohort. Neurosurgery 73:559-568, 2013

25. Trief PM, Grant W, Fredrickson B: A prospective study of psychological predictors of lumbar surgery outcome. Spine (Phila Pa 1976) 25:2616-2621, 2000

26. Vialle E, de Oliveira Pinto BM, Vialle LR, Gomez JDC: Evaluation of psychosomatic distress and its influence in the outcomes of lumbar fusion procedures for degenerative disorders of the spine. Eur J Orthop Surg Traumatol 25 (Suppl 1):S25-S28, 2015
27. Ware JE Jr: SF-36 health survey update. Spine (Phila Pa 1976) 25:3130-3139, 2000

\section{Disclosures}

Funding for the International Spine Study Group Foundation, through which this study was conducted, was provided by research grants from DePuy Spine and individual donations. Dr. Shaffrey is a consultant for Biomet, Medtronic, and Nuvasive; owns stock in Nuvasive; and is a patent holder with and receives royalty payments from Biomet, Medtronic, and Nuvasive. Dr. Smith is a consultant for Biomet, Nuvasive, and Cerapedics; received clinical or research support for this study from DePuy/ISSGF; receives support for other non-study-related clinical and/or research from DePuy/ISSGF; and receives royalties from Biomet. Dr. Hart is a consultant for DePuy Synthes and Globus; is a patent holder with OHSU; receives nonrelated grants from Medtronic; receives royalties from Seaspine and DePuy Synthes; is a board member of CSRS, ISSLS, and ISSG (has received no financial compensation to date); and performs speaking engagements for DePuy and Globus. Dr. Lafage has ownership interest and direct stock ownership in Nemaris INC; is a consultant for NuVasive; has speaking/ teaching arrangements with Medicrea, DePuy Spine, NuVasive, and Nemaris INC; and receives support of non-study-related clinical/research efforts she oversees from SRS, NIH, and DePuy Spine. Dr. Schwab has ownership of and direct stock ownership in Nemaris INC; is a consultant for K2M, NuVasive, Medicrea, Zimmer-Biomer, and MSD; is a patent holder for MSD and K2M; receives support for other non-study-related clinical/research efforts from AO, DePuy, and SRS; and has speaking/teaching arrangements with K2M, NuVasive, Medicrea, Zimmer-Biomer, and MSD. Dr. Burton received clinical/research support (equipment/material) from DePuy for this study and is a consultant and a patent holder for DePuy. Dr. Bess is a consultant for K2M and Allosource; is a patent holder for K2M and Innovasis; received clinical/research support for this study; and receives support for other non-study-related clinical/research efforts from Medtronic, Stryker, K2M, and Innovasis. Dr. Ames is a consultant for DePuy, Medtronic, and Stryker; has direct stock ownership in Doctors Research Group; is a patent holder for Fish \& Richardson, P.C.; and receives royalties from Biomet Spine and Stryker. Dr. Gupta is a consultant for DePuy and Medtronic; has direct stock ownership in Orthofix, Proctor \& Gamble, Pfizer, Johnson \& Johnson, and Pioneer; and receives royalties from DePuy. Dr. Klineberg is a consultant for DePuy Synthes and Stryker; and receives an honorarium from AO Spine and a grant for a fellowship from K2M.

\section{Author Contributions}

Conception and design: Theologis, Ames. Acquisition of data: Theologis. Analysis and interpretation of data: Theologis, Ailon. Drafting the article: Theologis, Ailon. Critically revising the article: all authors. Reviewed submitted version of manuscript: all authors. Approved the final version of the manuscript on behalf of all authors: Theologis. Statistical analysis: Ailon. Study supervision: Shaffrey, Bess, Gupta, Klineberg, Kebaish, Schwab, Lafage, Hart, Ames.

\section{Supplemental Information \\ Previous Presentations}

Portions of this work were presented as an oral presentation at the 22nd International Meeting on Advanced Spine Techniques (IMAST), Kuala Lumpur, Malaysia, July 8-11, 2015.

\section{Correspondence}

Alexander Theologis, Department of Orthopaedic Surgery, University of California, San Francisco, 500 Parnassus Ave., MU West 3rd Fl., San Francisco, CA 94143. email: alekos.theologis@ ucsf.edu. 